\title{
Integrating Clinical Practice and Research Skills into Comprehensive Capability for Translational Medicine, Zhejiang Experience on the Hepato-bilio-pancreatic Surgeon Program
}

\author{
Xinhua Chen ${ }^{1}$, Guoping Jiang ${ }^{1}$, Shengyong Yin ${ }^{1}$, Haiyang Xie ${ }^{1}$, Lin Zhou ${ }^{1}$, Shusen Zheng ${ }^{1}$
}

\begin{abstract}
A highly skilled surgical team relies on a standardized training program. This article presents a comprehensive description on the current status, problems, and strategies in Hepato-bilio-pancreatic surgery education reform in Zhejiang University School of Medicine (ZJU), China. A multiple-discipline joint training program was set up by integrating the basic laboratory and clinical research as well as routine clinical practice. Reform includes resident training in the Royal College of Surgeons of Edinburgh, curriculum resetting, surgery textbook rewriting, clinical teaching and training bases construction, teaching methods improvement, English reading, writing and reporting skills training and a research assignment. ZJU establishes rigorous processes for managing teaching quality and ensuring graduates promote evidence-based surgical practice. ZJU has built its leading position in the Hepato-Bilio-Pancreatic surgery in China. In 2011, 6 post-doctoral researchers, 52 Ph.D. students and 83 master students were enrolled in the 7year MD program and 8year Ph.D. MD/Ph.D. program. The admission scores have been listed as the top three of the 264 master degrees programs and 181 doctoral degrees programs in Zhejiang University, which ranks among the top three in all Chinese Universities. Historical experience and the advanced experience of Western countries are adopted as a reference to build up a surgical education system with Chinese characteristics to achieve a continuous, sound, and rapid development of surgical education in ZJU, China. However, there are differences relating to the healthcare systems of China and Western countries. These differences present major challenges for the internationalization of surgical education. This article may facilitate the process of surgical training development in a global context.
\end{abstract}

Keywords: medical education, basic research, scientific training, education reform

\section{Background}

Surgery education in the People's Republic of China was established similarly to the Soviet model that has been in operation since 1950. China, with its dense population, may have the largest number of medical institutions in the world.

\footnotetext{
${ }^{1}$ Department of General Surgery,

The First Affiliated Hospital, School of Medicine,

Zhejiang University, Zhejiang Province,

Hangzhou 310003, China.

Corresponding author:

Dr. Shusen Zheng, The First Affiliated Hospital, School of Medicine, Zhejiang University, 79 Qingchun Road, Hangzhou 310003, Zhejiang Province, China.

E mail: shusenzheng@zju.edu.cn
}

There are approximately 160 higher medical institutions for 2 million students and approximately 1,900,000 graduates in 2011 . Autonomous medical universities offer various medicinal programs. There are 3 year diploma, 5 year bachelor, 7 year master and 8 year MD/Ph.D programs co-existing in the medical education spectrum. The difference from Western countries is that the Chinese medical students get clinical medicine admission without a four-year undergraduate program.

We are now in the era of translational medicine which advocates raising questions from clinical work and pursuing in-depth study in basic research, to rapidly turn the results of basic scientific research into clinical application to improve healthcare (Wan et al., 
2012). According to the national long-term education reform and development plan in China (2010-2020), the objective of higher education in China is to improve the quality of higher education. The First Teaching Hospital of Zhejiang University is positioned to train medical personnel of the elite with an international vision and capability of independent innovation. Although the government has assigned special funding into medical faculty development, curriculum reforms and accreditation to improve medical education, there are still five major challenges in the current surgery education.

(1) The most prominent issue in surgery study is the difference between theory and practical studies. The traditional teaching model is also known as the "duck-feeding" style. Many medical lecturers begin work in a medical school immediately after graduation and few have either first-hand experience or involvement in clinical practice. The difference between theory and practical study can be a hindrance for further development of surgery education in China.

(2) The standard textbooks do not relate much in comparison to the old Chinese versions of textbooks; hence do not expose the students to advanced development of modern surgery. Lecturers put more emphasis on examoriented teaching and attention to new knowledge and skills. There is limited reference for clinicians on curriculum development. As a consequence, much practical skills and knowledge are not stated in the textbook and curricula.

(3) Deeply influenced by Confucius, surgery teaching is based mainly on instruction instead of proactive investigation by the students themselves. The students are good in remembering textbooks and successful in multiple choices questions but very weak in raising their own questions or resolving real problems. The students are not well trained in the sense of creativity, innovation and imagination.

(4) There is no research opportunity during internship and fellowship which may weaken the potential in an academic career. When medical graduate students become the backbone of the hospital, they cannot train their students effectively in academics. Continuity of this malicious cycle in the long run will weaken the surgery development and quality of healthcare.
(5) the Surgery MD and Ph.D. Programs in Zhejiang University aim to train strong, scientifically oriented surgeons who can perform surgeries and also lead research projects in a laboratory setting. We believe that laboratory experience is valuable and will contribute to graduate students entering an academic career. However, the decreased internship time and increased clinical training demands have raised a question of feasibility when including research experience into the program. Medical students focus on clinical practice, and are not fully prepared for academic achievement although many universities put the $\mathrm{SCl}$ publication as a compulsory requirement for diploma.

Traditional surgery training focuses on basic medicine and graduate students have to spend 3 years on basic sciences and 1 year on surgery textbook and classroom study. The student has less than one year in clinical skill training (most commonly 1 year rotation as bedside study in different departments in teaching hospitals). Very rarely can they get laboratory research training. The contradiction on the need of clinical experts and the lack of research training calls for a solution.

\section{Reform}

In order to respond to the challenges of translational medicine and to create a translational medical centre, surgery teaching reforms have been attempted in the First Teaching Hospital of Zhejiang University to modify teaching practices and to include approaches based on clinical problems, curriculum resetting, textbook compiling, lab rotation requirement and research assignments, introduction of international advanced methodology, adoption of useful teaching philosophy as well as new teaching methods, e.g. PBL (Problem Based Learning) teaching models and information technology (Aggarwal \& Darzi, 2011).

\section{The resident training in the Royal College of Surgeons of Edinburgh}

The core of our program is surgeon training with excellent clinical skills. To ensure the highest standards of surgical practice and surgical training, we register residents with the Royal College of Surgeons of Edinburgh for excellence and innovation in surgical practice. Our partnership with the Royal College of Surgeons of Edinburgh began in 2010 and every year, 20 residents register for training according to the requirements of the College. Young surgeons in our program grow in the 
College's wide range of international activities, events, courses and exams.

\section{Curriculum resetting}

We are trying to differentiate our programs to balance the needs of the different durations of medical degrees. Shorter degree courses for example, the 7 year medical program is set up for clinical skills training, aiming to provide our hospitals with qualified residents, whereas the 8 year MD/Ph.D. degree programs are for the scientists, specialists and academic leaders. We also offer step-by-step master and MD or Ph.D. programs aspiring to produce competent clinicians and researchers.

\section{The construction of clinical teaching and training bases}

Several million Renminbis (RMBs/Chinese Yuan) have been invested on the establishment of a clinical skills training centre. We have deviated from the previous rigid enrolment system based only on exam scores and instead, admissions are given to those who have excellent academic records and express strong research desires in the interview. We also emphasize the rotation examination assessment to carry out the clinical skills of medical operation competitions.

\section{Rewriting surgery textbooks}

We have invited famous academicians and academic experts to participate in re-writing of textbooks. Our surgery textbook series is designed with different teaching objectives for the five year, seven year and eight year graduate programs. Up till 2012, the textbook has had the second edition and over 30 medical universities across the country use our surgery textbook series.

\section{Teaching methods improvement}

In addition to textbook content updates, we also changed the old classroom theoretical teachings into adopting the five-in-one method, incorporating lectures, tutorials, selfdirected learning, question and answer sessions, and open discussions, alongside a practical aspect which supports social practice, on-site teaching, seminars and undergraduate mentorship programs. Most importantly, PBL teaching is applied.

\section{English reading, writing and reporting skills training}

We have lead students to have morning shift meetings, case discussions, and grand ward rounds in the English language for more than 20 years and have discussed over 15,000 cases. An English Journal Club has also been established, guiding students to read clinical and basic research papers. When we set up the Joint Liver Centre together with UCLA and the cooperation with other overseas institutes, we have had video-conferencing discussions on a regular basis to study difficult cases. Our collaboration with more than 20 medical centres such as University of Kiel, University of Western Australia, University of Virginia, University of Kobe, Japan, University of Hannover, etc., has also helped our graduate students to learn with an open mind and a wider exposure to international standards (Pollett \& Waxman, 2012). We have also accepted over 400 foreign students to do surgery and lab internships to strengthen the English teaching capability.

\section{Research assignment requirement}

We require our medical students to begin with a research fellowship during training (Adra et al., 2012). We provide clinical research, translational research, or basic research opportunities. In order to ensure students can conduct their research, we are adopting strategies such as reserving protected research time (1-3 years) and providing a seed grant. From 1998 to 2012, we have provided 15 years of SRTP (the Student Research Training Program) for undergraduates, graduates and medical students in surgery education. They participate in research under the direct supervision of our faculty and staff. The project based on small funding helps students to rationalize and set up strategies, methods and other aspects of biomedical research as research assistants. The on-site clinical studies or the laboratory bench work need not interfere with the clinical training. We allow our medical students to perform external clinical duties ("moonlighting") during their post-doctoral study period. Our records show that students who are asked to perform research work prove to be more likely to gain funding and hold academic positions in future clinical careers.

Of former students who have performed research work, 26 received NSFC funding (similar to NIH R01). Three were awarded Zhejiang Province 151 Medical Talents Project. Four academic backbones were recognized with the Young Scientist Award at International Conferences.

\section{Support on research training}

Hospitals have earmarked special funds for scientific research work and strong rewards for such research. We send 20-30 faculty for 
overseas training annually. We also invite 1020 world famous surgery experts to make presentations or surgical illustrations in our hospital. We open the surgery lab to students, provide them with research funding and encourage them to participate in scientific research to stimulate enthusiasm in their training.

\section{Innovation in surgery teaching}

We have a conducive environment for open discussions to inspire the students to develop new ideas. When studying liver transplantation, although former textbooks insisted on Milan criteria or UCLA criteria, we raised the question if the criteria fit the Chinese context of high hepatitis B prevalence. We then developed the Hangzhou Criteria as the liver transplantation standard in China. This innovation has significant impact in promoting the Chinese transplantation course. The Hangzhou Criteria is a good example on how we find the question in clinical work to address major clinical needs, then look for pathological and biochemical methods to solve the problems (Carlile, 2012).

\section{Outcome}

\section{The leading position in surgery education in China}

We have achieved desired results. In 2007 our team was recognized as the National Classical Example Course on Surgery. The department of general surgery was awarded as one of the National Key Disciplines. We set up the first Translational Medicine Centre in China in 2010. Our teaching team was awarded as the National Innovative Research Team. We have been approved by the credential committee of the Royal College of Surgeons of Edinburgh, UK as a surgery teaching and training base with international certification. We also set up the collaboration with UCLA in the United States and HK University in Hong Kong to ensure our surgery teaching and training achieves international standards. In the process, we have established a cooperative relationship with 20 centres in 16 countries. In October 2011, Dr. Shusen Zheng was recognized as a UCLA honorary professor in surgery. He is the first Medical Honorary Professor from the Chinese Mainland in the history of the UCLA. Our lab has been supported by the Health Ministry as the Transplantation Key Laboratory which marks our leading academic position.

\section{Teaching Hospital Growth}

Due to sustainable development strategies we are motivated to meet our potential. Up to 2012, our hospital grew rapidly as a comprehensive teaching hospital of Zhejiang University. We have 4,052 employees, over $100,000 \mathrm{~m}^{2}$ flat space, $240,000 \mathrm{~m}^{2}$ building area, 2,500 hospital beds, 2.68 million outpatients/year, 83,000 inpatients/year and 28,000 operations/year. Our teaching teams include 538 faculty members with senior professional titles. Of special note, we have 2 Academicians of the Chinese Academy of Engineering, 45 faculty are Ph.D. supervisors and 198 are Master's level supervisors.

As the teaching team of general surgery in year 2012 we have 131 surgeons altogether and among them are 34 professors or chief physicians, 42 associate professors or deputy chief physicians. In the department of General Surgery, there are 15 wards with 542 beds to perform over 8800 operations/year; in which the subsections are Ano-rectal Surgery: 3 wards 92 beds, 3000 operations/year; Vascular Surgery: 1 ward 27 beds, 450 operations/year; Gastrointestinal, breast and thyroid Surgery: 2 wards 85 beds, 2200 operations/year; Hepato-biliary \& Pancreatic Surgery: 6 wards 244 beds, 3000 operations/year; Liver Transplantation centre, 3 wards 94 beds, 100 liver transplants/year. After nearly 20 years of hard work, we continue to mark success in major advances in clinical and research work, especially in organ transplant and general surgery disciplines. We have reached the international advanced level.

\section{Current medical students in our postgraduate programs}

In the year 2011, 6 post-doctoral researchers, 52 Ph.D. students and 83 Master's students were enrolled in our 7 year MD program and 8 year Ph.D. MD/Ph.D. program. Admission scores have been listed as the top three of the 264 master degrees programs and 181 doctoral degrees programs in Zhejiang University which ranks among the top three Chinese Universities.

\section{Outcome of high impact SCl journal publications}

The successful laboratory and clinical research is responsible for the increase of publication. Students published 160 papers in high impact peer reviewed SCl journals such as Liver Transplantation among which 7 year MD students contributed over 50 papers (Figure 1). 
Figure 1: Publications of trainees in the ZJU Hepato-bilio-pancreatic surgery program

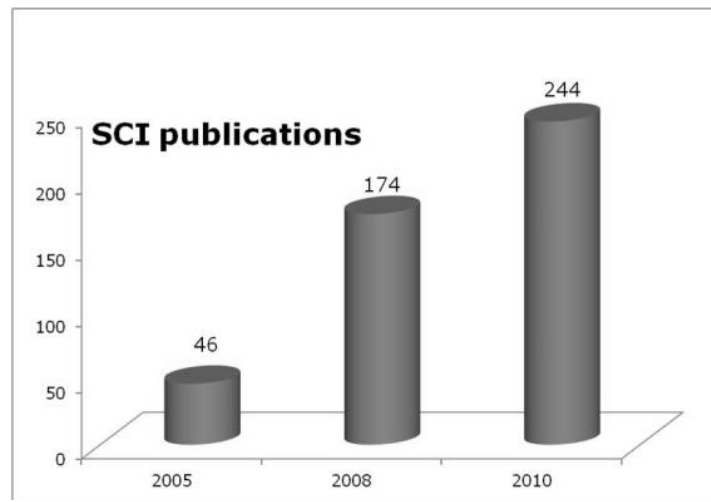

\section{The increase of the research funding and grants}

We have annual research fund in 2010: 245 Million RMB. The total research fund in (1999-2010) 700 Million RMBs (Figure 2). The teaching hospital has a total of 213 NSFC grants from National Science Fund of China. From 1999 to 2011 the grant number is steadily increasing. Our team has presided over two 973 projects (Chief Scientist), five National Science and Technology Support Programs (Figure 3).

\section{Figure 2: Increase of research funding}

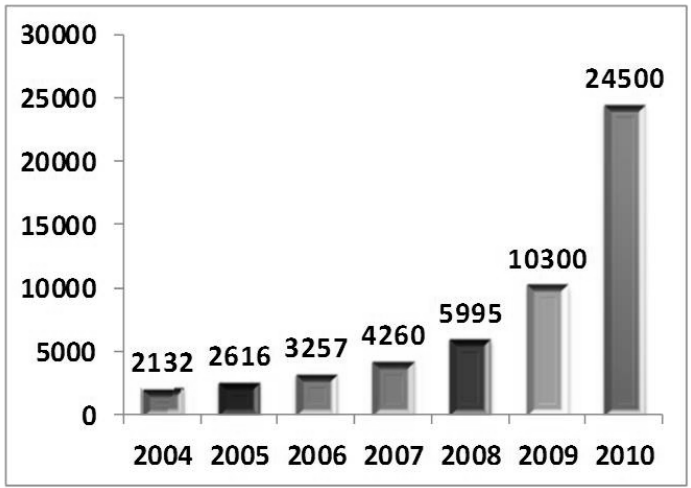

Figure 3: Increase of research grants

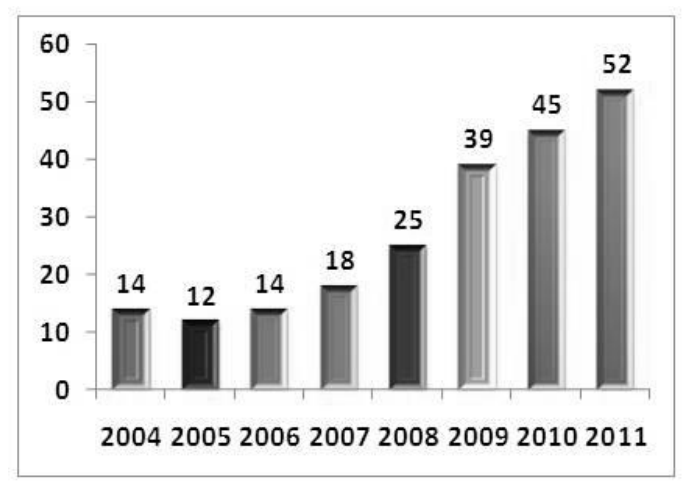

\section{Summary}

We are taking these actions to prepare our future surgeons to adequately meet high expectations and heavy responsibilities. We equip students to deal effectively with the rapid advances in modern surgery but also to adapt their laboratory training to a demanding academic career. We believe the success in medicine reform will provide good experiences to help direct the educational transformations and show positive effect on health care in China.

\section{Acknowledgements}

All authors thank Frank Reidy and Felicia Wong for kind comments and valuable inputs. The research is supported by Zhejiang University Teaching Reform Project, National S\&T Major Project (No. 2012ZX10002017), National Basic Research Program of China (973 Program) (No.2009CB522403 ), National Natural Science Foundation of China (No. 81372425), Zhejiang Medical Research Funding (No.2008B079, No.LY13H180003). SRF for ROCS, SEM (NO. J20120279).

\section{References}

Adra, S.W., Trickey, A.W., Crosby, M.E., Kurtzman, S.H., Friedell, M.L.\& Reines, H.D.(2012) General surgery vs fellowship: the role of the Independent Academic Medical Center, Journal of Surgical Education, 69, 6, pp.740-745.

Aggarwal, R. \& Darzi, A. (2011) Innovation in surgical education-a driver for change, Surgeon, vol.9, Suppl 1, pp.S30-S31.

Carlile, G.S. (2012) Teaching within the operating theater, Perspectives in Biology and Medicine, 55, pp.127-136.

Pollett, W.G. \& Waxman, B.P.(2012) Postgraduate surgical education and training in Canada and Australia: each may benefit from the other's experiences, ANZ Journal of Surgery, 82, 9, pp.581-587.

Wan, D.C., Wang, K.C. \& Longaker, M.T. (2012) Training the contemporary surgeon-scientist, Plastic and Reconstructive Surgery, 129, 4, pp.1023-1025. 